\title{
A genome scan for Plasmodium falciparum malaria identifies quantitative trait loci on chromosomes 5q31, 6p21.3, 17p12, and 19p13
}

\author{
Audrey Brisebarre ${ }^{1,2 \dagger}$, Brice Kumulungui ${ }^{3 \dagger}$, Serge Sawadogo ${ }^{4 \dagger}$, Alexandre Atkinson ${ }^{1,2}$, Séverine Garnier ${ }^{1,2}$,
} Francis Fumoux 2,5 and Pascal Rihet ${ }^{1,2^{*}}$

\begin{abstract}
Background: Genome-wide studies have mapped several loci controlling Plasmodium falciparum mild malaria and parasitaemia, only two of them being significant at the genome level. The objective of the present study was to identify malaria resistance loci in individuals living in Burkina Faso.

Methods: A genome scan that involved 314 individuals belonging to 63 families was performed. Markers located within chromosomes 6p21.3 and 17p12 were genotyped in 247 additional individuals belonging to 55 families. The linkage and the association of markers with parasitaemia and mild malaria were assessed by using the maximum-likelihood binomial method extended to quantitative trait linkage and the quantitative trait disequilibrium test, respectively.

Results: Multipoint linkage analysis showed a significant linkage of mild malaria to chromosome 6p21.3 (LOD score $3.73, P=1.710^{-5}$ ), a suggestive linkage of mild malaria to chromosome 19p13.12 ( $L O D$ score $2.50, P=3.510^{-4}$ ), and a suggestive linkage of asymptomatic parasitaemia to chromosomes 6p21.3 (LOD score 2.36, P=4.9 10 ${ }^{-4}$ ) and 17p12 ( $L O D$ score 2.87, $\mathrm{P}=1.410^{-4}$ ). Genome-wide family-based association analysis revealed a significant association between three chromosome $5 \mathrm{q} 31$ markers and asymptomatic parasitaemia, whereas there was no association with mild malaria. When taking into account 247 additional individuals, a significant linkage of asymptomatic parasitaemia to chromosome 17p12 (LOD score 3.6, $\mathrm{P}=210^{-5}$ ) was detected.

Conclusion: A new genome-wide significant malaria locus on chromosome 17p12 and a new suggestive locus on chromosome 19p13.12 are reported. Moreover, there was evidence that confirmed the influence of chromosomes $5 q 31$ and 6p21.3 as loci controlling mild malaria or asymptomatic parasitaemia.
\end{abstract}

Keywords: Plasmodium falciparum, Mild malaria, Parasitemia, Genome wide scan, Microsatellite markers, Linkage, Genetic association

\section{Background}

Malaria remains a major problem of public health in over one hundred countries worldwide. According to the World Health Organization (WHO), the number of cases in 2010 reached 219 millions (95\% CI 154-289 millions), among which over 660000 (95\% CI 490 000-836 000) were fatal [1]. The disease ranges from asymptomatic

\footnotetext{
*Correspondence: pascal.rihet@univ-amu.fr

${ }^{\dagger}$ Equal contributors

'INSERM, UMR1090 TAGC, Marseille F-13288, France

${ }^{2}$ Aix-Marseille Université, Marseille F-13288, France

Full list of author information is available at the end of the article
}

parasitaemia or mild malaria, to severe anaemia, severe respiratory distress, or cerebral malaria. Fatal outcome occurs nearly exclusively in patients infected with Plasmodium falciparum who progress to severe malaria. Many studies provide evidence for human genetic factors controlling the outcome of infection by P. falciparum [2]. In particular, several candidate genes have been associated with resistance against severe malaria, while two GWA studies confirmed the $H B B$ locus as a major locus in $P$. falciparum severe malaria [3,4]. Linkage analyses pointed out some significant linkage on chromosomes 6p21-p23 and 10p15, and several suggestive linkage 
with mild malaria or parasitaemia on chromosomes $1 \mathrm{p} 36$, 2p25, 4q13-q21, 5p15-p13, 5q31-q33, 6p25.1, 6q15-q16, 9q34, 12q21-q22, 13q13, 20p12 and 20q11 [5-9]. It should be stressed that linkage of mild malaria to chromosome 6p21-p23 and linkage of asymptomatic parasitaemia to 5q31-q33 have been reported at least twice in humans; these two loci correspond respectively to Char3 and Char8 that control Plasmodium chabaudi in mice $[10,11]$.

The present study reports results from a genome-wide scan and additional further testing of promising regions. The maximum-likelihood binomial method extended to quantitative trait linkage analysis (MLB-QTL) and the quantitative trait disequilibrium test (QTDT) were applied to search for genetic linkage and association in the presence of linkage with mild malaria and asymptomatic parasitaemia.

\section{Methods}

\section{Subjects and phenotype determination}

The initial study population consisted of 314 individuals belonging to 63 families living in an urban district of Bobo Dioulasso in Burkina Faso; the mean age of sibs was $13.6 \pm 6.3$ years (range 1-34 years). The additional study subjects live in a rural area, Logoforousso, a village to the south-west of Bobo-Dioulasso (Burkina Faso). The study population comprised 247 subjects from 55 nuclear families; the mean age of the sibs was $10.1 \pm 4.7$ (3-29 years). The populations and the areas of parasite exposure have been extensively described $[7,12]$. Informed consent was obtained individually from all participants or their parents. The protocol was approved by the regional and national medical authorities of Burkina Faso. Parasitaemia data and DNA were available for the initial and additional study subjects. In addition, febrile episodes were extensively recorded by active case detection during 24 months for the initial population. For patients with fever, a thick blood film was prepared by the standard procedures. Diagnosis of mild malaria attack was based on $P$. falciparum parasitaemia, fever (axillary temperature more than $37.5^{\circ} \mathrm{C}$ ) and clinical symptoms (headache, aching, vomiting or diarrhoea in the children); in that case no threshold of parasitaemia was used. In the absence of classical symptoms of malaria, and once others pathologies could not be eliminated, only children (age $<15$ years) with more than 5,000 parasites per $\mu \mathrm{l}$ and older subjects with more than 2,000 parasites per $\mu \mathrm{l}$ were considered as having had a malaria attack. Each episode of illness was treated according to the recommendation of the CNRFP (Centre National de Recherche et Formation sur le Paludisme) of Burkina Faso. Parasitaemia was checked at the end of the treatment. Subjects who presented at least one mild malaria attack during the survey were considered in the analysis affected, while the others were considered unaffected.
Determination of parasitaemia was described in previous studies [7,12]. Briefly, each family was visited 20 times during the 24 months of the study in the initial population, and 24 times during 24 months in the additional one, and parasitaemia was measured. In addition, parasitaemia was measured during febrile episodes. The mean number of parasitaemia measurements per subject was $15.2 \pm 5.1$. Fingerprint peripheral blood samples were taken from all family members present and thick and thin blood films were stained with Giemsa. The parasite determination and numeration were established blindly from two independent readings. Only $P$. falciparum asexual forms were retained to determine parasitaemia. Parasitaemia was defined as the number of parasitized erythrocytes observed per $\mu \mathrm{l}$ in thin blood films.

Mean of adjusted asymptomatic parasitaemia was a logarithmic transformation of the parasitaemia adjusted for seasonal transmission [7,12], after excluding parasitaemia during febrile episodes. To take into account the seasonality of the transmission, the influence of the date of the visits on $\ln (1+$ parasitaemia) (LP) was evaluated by one way analysis of variance. The mean LP observed during each visit was calculated. The individual LP was then corrected for the visit effect by subtracting from each individual LP the mean LP of the corresponding visit. Phenotypes were calculated using the SPSS software (SPSS, Boulogne, France). Logistic regression and linear regression analyses confirmed the effect of age on mild malaria and mean of adjusted asymptomatic parasitaemia, respectively. Logistic regression analysis that took into account the effect of age also showed a positive correlation between mild malaria and asymptomatic parasitaemia $(P=0.013)$. The residual of the logistic regression model and the one of the linear regression model were calculated for mild malaria and parasitaemia, respectively; the residuals were further used in linkage or association analyses. All the sibs (in full) were included in linkage analyses.

\section{Genotyping}

Genome scan was performed by using 400 microsatellite markers (Panel MD-10, Applied Biosystems), with an average distance of $10 \mathrm{cM}$. Multiplex PCR was performed under standard conditions, and the products were analysed by using a ABI377 systems (Applied Biosystems). Additional genotypes that were previously characterized in the initial study population were used for chromosomes 6p21.3 (TNFb, TNFd, D6S291) and 5q31-q33 (D5S642, D5S2117, D5S393, D5S399, D5S658, D5S436, D5S2090, D5S636, D5S2012, and D5S487) [5,7]. Additional markers were genotyped in the initial population comprising 314 individuals for chromosomes 5q31-q33 (D5S490, IRF1 microsatellite, IL4 microsatellite, IL9 microsatellite, 
D5S2115, D5S2017, D5S1972, ADRB2 microsatellite, D5S673, D5S410, and IL12B microsatellite) and 17p12 (D17S1881, D17S1844, D17S1812, D17S1791, D17S945, D17S1875, D17S969, D17S1856, D17S839, D17S953, D17S1824, D17S1800, D17S1850, D17S933, and D17S946). Additional markers were genotyped in the additional study population comprising 247 individuals for chromosomes 6p21.3 (TNFb, TNFd, D6S291) and 17p12 (D17S1881, D17S1844, D17S1812, D17S1791，D17S945, D17S1875, D17S969, D17S1856, D17S839, D17S953, D17S1824, D17S1800, D17S1850, D17S933, and D17S946). Genotyping procedures have been described for $6 \mathrm{p} 21.3$ and 5q31-q33 microsatellite markers [5,12]. The additional microsatellite markers located on chromosome $17 \mathrm{p} 12$ were genotyped under standard conditions. Mendelian inconsistencies were checked using the PedCheck program [13], whereas unlikely genotypes were detected using the MERLIN program [14]. Genotypes creating inconsistencies were corrected after re-examining raw data and re-genotyping if necessary.

\section{Statistical analyses}

Nonparametric multipoint linkage analyses were performed with the MLBGH 3.0 program, which uses the general framework of Genehunter program $[15,16]$. The maximum likelihood binomial (MLB) method, which is based on the binomial distribution of parental marker alleles among affected offspring, overcomes the common problem of multiple sibs by considering the sibship as a whole. The MLB method in model free linkage analysis of quantitative traits was used to assess linkage of the residuals to genetic markers. The quantitative trait method introduces a latent binary variable $(0 ; 1)$ that captures the linkage information between the observed quantitative trait and the marker. The fully non-parametric approach with no assumption on the distribution of the residual for mild malaria was applied. The residual values were divided into 10 consecutive equal subintervals with equal probabilities. The probability to have a 0 value was fixed at $0.95,0.85,0.75,0.65,0.55,0.45,0.35,0.25,0.15$ and 0.05 for phenotypes belonging to the consecutive deciles (1-10). The approach with the assumption on the normal distribution was used for parasitaemia. The MLB statistics was expressed in terms of a MLB-LOD and a one-sided standard normal deviate, denoted ZMLB. According to Kruglyak and Lander recommendations [17], linkage was considered suggestive and significant when the LOD score was higher than 2.2 and 3.6, respectively. The former is defined as the LOD score that would expected to occur one time by chance in a whole genome scan; the later is defined as the LOD score that would expected to occur 0.05 times by chance in a whole genome scan.
Combined association and linkage analyses of quantitative traits were carried out using the orthogonal model released in the QTDT 2.6.1 program [18]. Variance components are used to construct a test that utilizes information from all available offspring. The orthogonal model partitions association into between and within family components, and allowed use of markers and phenotypes as covariates in analyses. Evidence of association can be evaluated by likelihood-ratio test (null hypothesis likelihood L0 vs alternative hypothesis likelihood L1). Asymptotically, the quantity 2(LnL1-LnL0) is distributed as $\chi^{2}$ with df equal to the difference in number of parameters estimated. A false discovery rate of $10 \%$ was applied to correct for multiple testing [19].

\section{Results}

Genomewide linkage or association analysis of mild malaria and asymptomatic parasitaemia

The genome scan was performed on 314 individuals belonging to 63 families. Figure 1 shows the multipoint LOD scores obtained for mild malaria. LOD scores indicating suggestive (LOD score $>2.2$ ) or significant (LOD score $>3.6$ ) linkage were achieved at 2 positions on chromosomes $6 \mathrm{p} 21.3$ and 19p13. A significant genome-wide LOD score and a suggestive genome-wide LOD score were obtained for mild malaria on chromosome $6 \mathrm{p} 21.3$ (LOD score $=3.73, \mathrm{P}=1.710^{-5}$ ) and on chromosome 19p13 (LOD score $=2.50, \mathrm{P}=3.510^{-4}$ ), respectively. The 1-LOD drop area for QTL location on chromosome 6 p21.3 was very small, and included only 5 genes: $T N F$, LTA, LTB, LST1, and NCR3.

Figure 2 shows the LOD scores obtained for asymptomatic parasitaemia. Initial multipoint analyses revealed suggestive linkage on two chromosomes. For the $6 \mathrm{p} 21.3$ region that was significantly linked to mild malaria, we detected a suggestive linkage to asymptomatic parasitaemia $\left(\right.$ LOD score $=2.36, P=4.910^{-4}$ ); the 1 -LOD drop area for QTL location was very similar than the one for mild malaria, and contained the same genes. The best LOD score was achieved on chromosome $17 \mathrm{p} 12$ at D17S969/D17S799 (LOD score $\left.=2.87, \mathrm{P}=1.410^{-4}\right)$. The 1-LOD drop area for QTL location on chromosome 17p12 spanned 14 cM (D17S1875-D17S839), and contained 11 genes: MAP2K4, ARHGAP44, MYOCD, ELAC2, COX10, DNAH9, HS3ST3B1, HS3ST3A1, ZNF18, CDRT15, and SHISA6.

Combined linkage and association between malaria phenotypes and microsatellite markers were further tested. After applying a FDR of $10 \%$, there was no significant association with mild malaria, whereas there was an association of asymptomatic parasitaemia with D5S642, the IL9 microsatellite, and D5S2017. Strikingly, those genetic markers are located within chromosome 5q31; noticeably, the distance between D5S642 and the IL9 


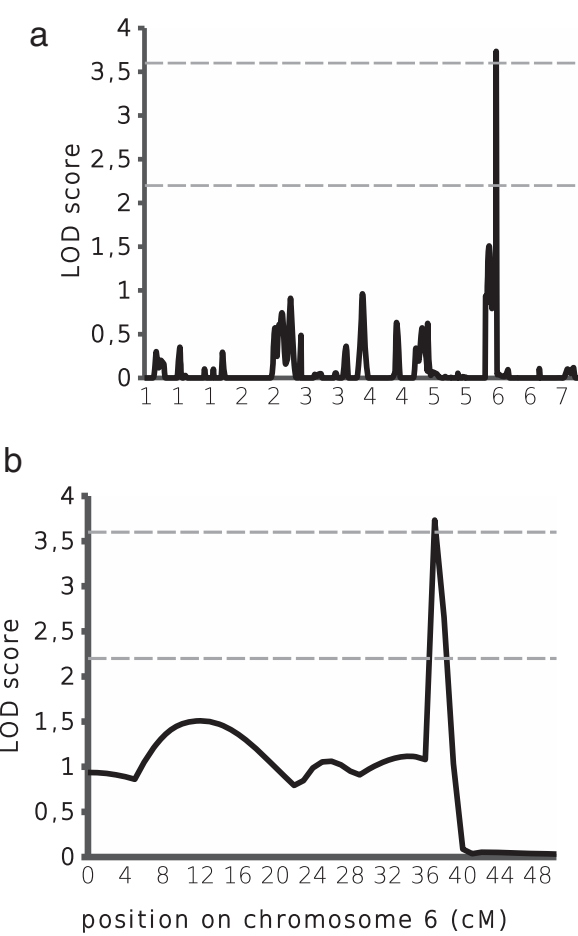

Figure 1 Genomewide scan results of mild malaria. A total of 314 individuals belonging to 63 nuclear families had been included in the analysis. Multipoint LOD scores implemented by MLBGH were plotted along the 22 autosomes (a). The horizontal lines represent the threshold for a suggestive linkage $(L O D$ score $=2.2)$ and a significant linkage $(L O D$ score $=3.6)$. Detailed view of the regions with the highest $L O D$ score on chromosomes 6 (b) and 19 (c).

microsatellite was $3.7 \mathrm{cM}$, and the one between the IL9 microsatellite and D5S2017 was $6.7 \mathrm{cM}$, suggesting the existence of several distinct malaria resistance loci in this region.

\section{Linkage of asymptomatic parasitaemia to chromosomes $17 p 12$ and 6p21.3}

Additional individuals $(\mathrm{n}=247)$ that belonged to 55 families were also genotyped for the microsatellite markers in chromosomes $6 \mathrm{p} 21.3$ and $17 \mathrm{p} 12$, resulting in a total of 118 families with 561 individuals genotyped. The additional genotyping led to higher LOD scores for chromosomes 6p21.3 (LOD score $=3.16, \mathrm{P}=7 \quad 10^{-5}$ ) and $17 \mathrm{p} 12$ (LOD score $=3.6, \mathrm{P}=210^{-5}$ ), as shown in Figure 2. The whole 1-LOD drop area for QTL location on chromosome 6p21.3 contained 5 genes: TNF, LTA, LTB, LST1, and NCR3. The whole 1-LOD drop area for QTL location on chromosome 17p12 spanned 13 cM (D17S969D17S839), and contained the 11 genes identified in the primary analysis.

\section{Discussion}

Genome-wide linkage and association approaches were used to identify loci controlling parasitaemia or mild malaria. Four hundred thirty three microsatellite markers were genotyped for 314 individuals belonging to 63 families, and genome-wide analyses were performed to assess linkage and association between markers and malaria phenotypes that were monitored for two years. Additional markers located within chromosomes 6p21.3 and $17 \mathrm{p} 12$ and genotyped in 247 individuals belonging to 55 families were included in the analysis to further assess the linkage of asymptomatic parasitaemia to those chromosomal regions. The linkage and association results point out the influence of chromosomes $6 \mathrm{p} 21.3$, $19 \mathrm{p} 13,5 \mathrm{q} 31$, and $17 \mathrm{p} 12$ on mild malaria or asymptomatic parasitaemia.

The linkage results strongly support chromosome 6 p21.3 as a locus controlling mild malaria, and suggest the influence of the same locus on parasitaemia. It should be stressed that the linkage of mild malaria to chromosome 6 p21.3 was not a replicating study, because it mostly corresponds to the study published [5]. However, the suggestive linkage of parasitaemia to chromosome $6 \mathrm{p} 21.3$ was a new result, and strengthens the result previously obtained. These results are in line with results obtained in an independent human population [20] and mice [21]. Noticeably, the 1-LOD drop area contained only five genes, and among them, TNF, LTA, and NCR3 have been associated with mild malaria or parasitaemia [22-24]. Besides, the results suggest a linkage of mild malaria to chromosome 19p13. The 1-LOD 


\section{a}

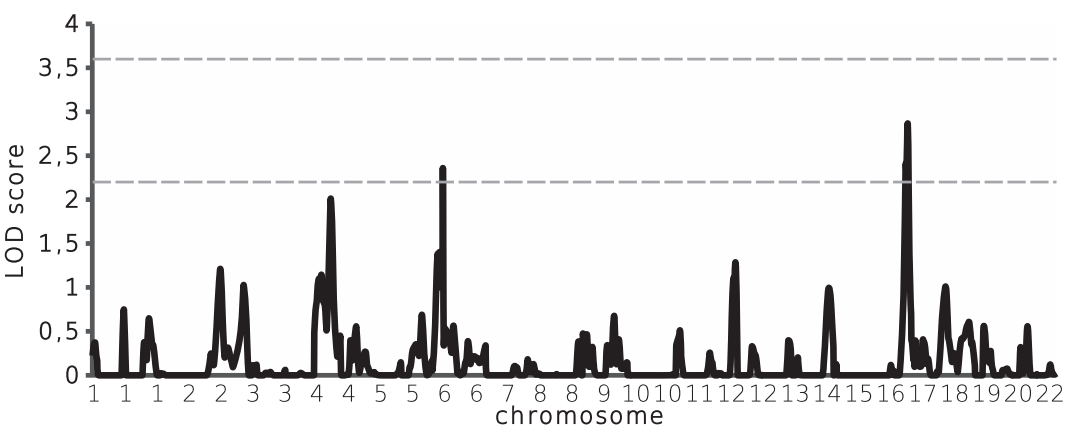

b

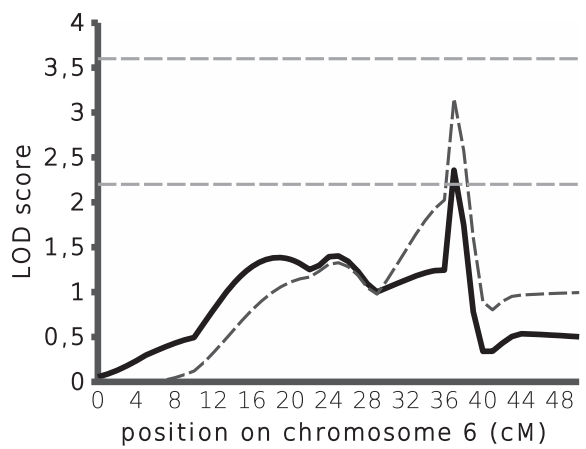

c

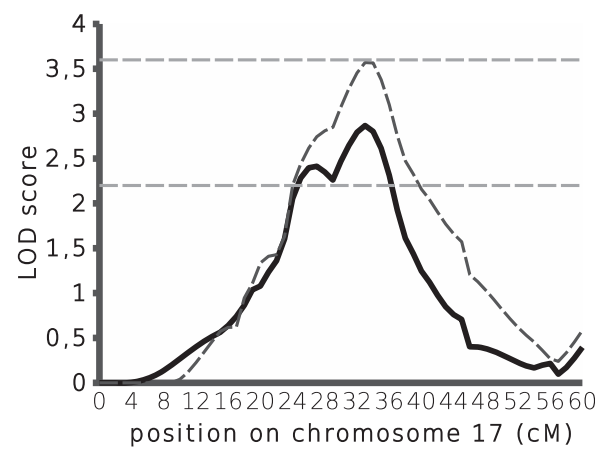

Figure 2 Genomewide scan results of asymptomatic parasitaemia. Multipoint LOD scores implemented by MLBGH were plotted along the 22 autosomes (a). The horizontal lines represent the threshold for a suggestive linkage ( $L O D$ score $=2.2$ ) and a significant linkage ( $L O D$ score $=$ 3.6). Detailed view of the regions with the highest LOD score on chromosomes 6 (b) and 17 (c). The primary genome scan results were based on 314 individuals belonging to 63 nuclear families (solid lines). The extended linkage results were based on 247 additional individuals belonging to 55 nuclear families, resulting in 561 individuals belonging to 118 families (dotted lines).

drop area for QTL location on chromosome 19p13 contained 233 genes. Interestingly, chromosome 19p13 has been also found to be associated with severe malaria [3], whereas it has not been reported as a mild malaria locus so far.

The influence of chromosome $5 \mathrm{q} 31$, which has been reported $[6-8,12,25,26]$, is further supported by our linkage and association results at the genome wide significance level in the initial population. It should be stressed, however, that the linkage analysis did show neither a significant nor a suggestive linkage between asymptomatic parasitaemia and chromosome 5q31-q33; it provided evidence for two small peaks of linkage, yet with an LOD score lower than 2.2, whereas only one peak of linkage has been detected in previous linkage studies $[7,8,12]$. In particular, the previous linkage analysis that was performed in the same population by using another statistical method yielded a large peak of linkage, which may correspond at least to 2 loci showing a weak linkage signal in the present study [7]. In the same way, the genome-wide linkage and association analysis yielded a significant association of asymptomatic parasitaemia with three chromosome $5 \mathrm{q} 31$ microsatellite markers, which were several $\mathrm{cM}$ distal to each other, suggesting the existence of several malaria resistance loci on chromosome $5 \mathrm{q} 31$. This is consistent with the association study performed in the additional population [12], although the genetic markers associated with parasitaemia in the additional population and those associated with parasitaemia in the initial population were different. This is also consistent with linkage analyses in mice [11] and genetic studies in humans showing an association of IL3, IL4, IL13, IRF1, and ARHGAP26 with parasitaemia, mild malaria or severe malaria [6,27-30].

Finally, a new locus controlling parasitaemia was identified based on a genome-wide significant LOD score after adding new families in the study. The peak of linkage located within chromosome $17 \mathrm{p} 12$ was sharp, and the 1-LOD drop area contained 11 genes both in the initial population and the extended one, the mouse orthologs of which are within Char8 controlling parasitaemia in mice infected by $P$. chabaudi [11]. Among them, HS3ST3A1 and HS3ST3B1 were considered candidate genes on the basis of their function and the location of their mouse orthologs within Char8, and were found to be associated with asymptomatic parasitaemia in a population that corresponded mostly to the additional population in the present study [31]; interestingly, the linkage analysis that was performed in the initial population provided a suggestive linkage of parasitaemia to chromosome 17p12. HS3ST3A1 and HS3ST3B1 encode 3-OST- 
3A1 and 3-OST-3B1, respectively, which are sulphotransferases involved in heparan sulphate (HS) synthesis. They catalyse the 3-0 sulfation of carbohydrates, which is the last step of the synthesis, and which reflects the completion of HS synthesis. The variation in human genes controlling the sulphation of HS may alter the binding of sporozoites to hepatocytes and their development in those cells, as it has been shown in mice infected by $P$. berghei [32]. Taken together, all these results suggest that the genes involved in HS synthesis and located on chromosome $17 \mathrm{p} 12$ play a major role in the control of parasitaemia. Alternatively, other genes located in the same chromosomal region may also influence parasitaemia, and the additive effect of several genes may explain the strong linkage of parasitaemia to chromosome $17 \mathrm{p} 12$.

This is the first report of a malaria resistance locus on chromosome $17 \mathrm{p} 12$, whereas it confirms previous loci on chromosomes $6 \mathrm{p} 21.3$ and $5 \mathrm{q} 31$. Although replication studies are needed, our results support the recent hypothesis that genetic variation within genes involved in HS synthesis strongly influences parasitaemia. This may provide new insights for developing new therapeutic strategies.

\section{Competing interests}

The authors declare that they have no competing interests.

\section{Authors' contributions}

$A B$ carried out the linkage and association analyses. BK and SW genotyped the microsatellite markers, and constructed the genotype database. AA and SG participated in genotyping. FF participated in the design of the study, and revised the results and the manuscript. PR performed the design of the study, supervised the experiments and the statistical analyses, participated in the statistical analyses and the interpretation of data, and wrote the manuscript. All authors read and approved the final manuscript.

\section{Acknowledgments}

This work was supported by grants from the Centre National de Genotypage, the French ministry, and Institut National de la Santé et de la Recherche Médicale (Inserm). AB and SG were supported by a PhD fellowship from the French ministry and a PhD fellowship from the PACA council and Inserm, respectively. We thank all the individuals for participating in the study. We also thank the Centre National de Genotypage for providing access to their genotyping facilities.

\section{Author details}

${ }^{1}$ INSERM, UMR1090 TAGC, Marseille F-13288, France. ${ }^{2}$ Aix-Marseille Université, Marseille F-13288, France. ${ }^{3}$ Université des Sciences et Techniques de Masuku, Institut National Supérieur d'Agronomie et de Biotechnologies, Franceville, Gabon. ${ }^{4}$ University of Ouagadougou, UFR des Sciences de la Santé, Ouagadougou, Burkina-Faso. ${ }^{5}$ UMR-MD3, Marseille F-13385, France.

Received: 7 February 2014 Accepted: 20 May 2014

Published: 28 May 2014

\section{References}

1. WHO: World malaria report 2012. Geneva: World Health Organization 2012:288.

2. Kwiatkowski DP: How malaria has affected the human genome and what human genetics can teach us about malaria. Am J Hum Genet 2005, 77:171-192.

3. Jallow M, Teo YY, Small KS, Rockett KA, Deloukas P, Clark TG, Kivinen K, Bojang KA, Conway DJ, Pinder M, Sirugo G, Sisay-Joof F, Usen S, Auburn S, Bumpstead SJ, Campino S, Coffey A, Dunham A, Fry AE, Green A,
Gwilliam R, Hunt SE, Inouye M, Jeffreys AE, Mendy A, Palotie A, Potter S, Ragoussis J, Rogers J, Rowlands K, et al: Genome-wide and fine-resolution association analysis of malaria in West Africa. Nat Genet 2009, 41:657-665.

4. Timmann C, Thye T, Vens M, Evans J, May J, Ehmen C, Sievertsen J, Muntau B, Ruge G, Loag W, Ansong D, Antwi S, Asafo-Adjei E, Nguah SB, Kwakye KO, Akoto AO, Sylverken J, Brendel M, Schuldt K, Loley C, Franke A Meyer CG, Agbenyega T, Ziegler A, Horstmann RD: Genome-wide association study indicates two novel resistance loci for severe malaria. Nature 2012, 489:443-446.

5. Flori L, Sawadogo S, Esnault C, Delahaye NF, Fumoux F, Rihet P: Linkage of mild malaria to the major histocompatibility complex in families living in Burkina Faso. Hum Mol Genet 2003, 12:375-378.

6. Milet J, Nuel G, Watier L, Courtin D, Slaoui Y, Senghor P, Migot-Nabias F, Gaye O, Garcia A: Genome wide linkage study, using a 250K SNP map, of Plasmodium falciparum infection and mild malaria attack in a Senegalese population. PLoS One 2010, 5:e11616.

7. Rihet P, Traore Y, Abel L, Aucan C, Traore-Leroux T, Fumoux F: Malaria in humans: Plasmodium falciparum blood infection levels are linked to chromosome 5q31-q33. Am J Hum Genet 1998, 63:498-505.

8. Sakuntabhai A, Ndiaye R, Casademont I, Peerapittayamongkol C, Rogier C, Tortevoye P, Tall A, Paul R, Turbpaiboon C, Phimpraphi W, Trape JF, Spiegel A, Heath S, Mercereau-Puijalon O, Dieye A, Julier C: Genetic determination and linkage mapping of Plasmodium falciparum malaria related traits in Senegal. PLoS One 2008, 3:e2000.

9. Timmann C, Evans JA, Konig IR, Kleensang A, Ruschendorf F, Lenzen J, Sievertsen J, Becker C, Enuameh Y, Kwakye KO, Opoku E, Browne EN, Ziegler A, Nürnberg P, Horstmann RD: Genome-wide linkage analysis of malaria infection intensity and mild disease. PLoS Genet 2007, 3:e48.

10. Hernandez-Valladares M, Naessens J, Gibson JP, Musoke AJ, Nagda S, Rihet P, Ole-MoiYoi OK, Iraqi FA: Confirmation and dissection of QTL controlling resistance to malaria in mice. Mamm Genome 2004, 15:390-398.

11. Hernandez-Valladares M, Rihet $P$, ole-MoiYoi OK, Iraqi FA: Mapping of a new quantitative trait locus for resistance to malaria in mice by a comparative mapping approach with human chromosome 5q31-q33. Immunogenetics 2004, 56:115-117.

12. Flori L, Kumulungui B, Aucan C, Esnault C, Traore AS, Fumoux F, Rihet P: Linkage and association between Plasmodium falciparum blood infection levels and chromosome 5q31-q33. Genes Immun 2003, 4:265-268.

13. O'Connell JR, Weeks DE: PedCheck: a program for identification of genotype incompatibilities in linkage analysis. Am J Hum Genet 1998, 63:259-266.

14. Abecasis GR, Cherny SS, Cookson WO, Cardon LR: Merlin-rapid analysis of dense genetic maps using sparse gene flow trees. Nat Genet 2002, 30:97-101.

15. Alcais A, Abel L: Maximum-Likelihood-Binomial method for genetic model-free linkage analysis of quantitative traits in sibships. Genet Epidemiol 1999, 17:102-117.

16. Kruglyak L, Daly MJ, Reeve-Daly MP, Lander ES: Parametric and nonparametric linkage analysis: a unified multipoint approach. Am J Hum Genet 1996, 58:1347-1363.

17. Lander E, Kruglyak L: Genetic dissection of complex traits: guidelines for interpreting and reporting linkage results. Nat Genet 1995, 11:241-247.

18. Abecasis GR, Cardon LR, Cookson WO: A general test of association for quantitative traits in nuclear families. Am J Hum Genet 2000, 66:279-292.

19. Benjamini Y, Drai D, Elmer G, Kafkafi N, Golani I: Controlling the false discovery rate in behavior genetics research. Behav Brain Res 2001, 125:279-284.

20. Jepson A, Sisay-Joof F, Banya W, Hassan-King M, Frodsham A, Bennett S, Hill AV, Whittle H: Genetic linkage of mild malaria to the major histocompatibility complex in Gambian children: study of affected sibling pairs. BMJ 1997, 315:96-97.

21. Hernandez-Valladares M, Naessens J, Iraqi FA: Genetic resistance to malaria in mouse models. Trends Parasitol 2005, 21:352-355.

22. Barbier M, Delahaye NF, Fumoux F, Rihet P: Family-based association of a low producing lymphotoxin-alpha allele with reduced Plasmodium falciparum parasitemia. Microbes Infect 2008, 10:673-679.

23. Delahaye NF, Barbier M, Fumoux F, Rihet P: Association analyses of NCR3 polymorphisms with $P$. falciparum mild malaria. Microbes Infect 2007, 9:160-166.

24. Flori L, Delahaye NF, Iragi FA, Hernandez-Valladares M, Fumoux F, Rihet P: TNF as a malaria candidate gene: polymorphism-screening and 
family-based association analysis of mild malaria attack and parasitemia in Burkina Faso. Genes Immun 2005, 6:472-480.

25. Garcia A, Marquet S, Bucheton B, Hillaire D, Cot M, Fievet N, Dessein AJ, Abel L: Linkage analysis of blood Plasmodium falciparum levels: interest of the 5q31-q33 chromosome region. Am J Trop Med Hyg 1998, 58:705-709.

26. Naka I, Nishida N, Patarapotikul J, Nuchnoi P, Tokunaga K, Hananantachai $H$, Tsuchiya N, Ohashi J: Identification of a haplotype block in the $5 \mathrm{q} 31$ cytokine gene cluster associated with the susceptibility to severe malaria. Malar J 2009, 8:232.

27. Mangano VD, Luoni G, Rockett KA, Sirima BS, Konate A, Forton J, Clark TG, Bancone G, Sadighi Akha E, Kwiatkowski DP, Modiano D: Interferon regulatory factor-1 polymorphisms are associated with the control of Plasmodium falciparum infection. Genes Immun 2008, 9:122-129.

28. Meyer CG, Calixto Fernandes MH, Intemann CD, Kreuels B, Kobbe $\mathrm{B}$, Kreuzberg C, Ayim M, Ruether A, Loag W, Ehmen C, Adjei S, Adjei O, Horstmann RD, May J: IL3 variant on chromosomal region 5q31-33 and protection from recurrent malaria attacks. Hum Mol Genet 2011, 20:1173-1181

29. Ohashi J, Naka I, Patarapotikul J, Hananantachai H, Looareesuwan S, Tokunaga K: A single-nucleotide substitution from $\mathrm{C}$ to $\mathrm{T}$ at position -1055 in the IL-13 promoter is associated with protection from severe malaria in Thailand. Genes Immun 2003, 4:528-531.

30. Cabantous S, Poudiougou B, Oumar AA, Traore A, Barry A, Vitte J, Bongrand P, Marquet S, Doumbo O, Dessein AJ: Genetic evidence for the aggravation of Plasmodium falciparum malaria by interleukin 4. J Infect Dis 2009, 200:1530-1539.

31. Atkinson A, Garnier S, Afridi S, Fumoux F, Rihet P: Genetic variations in genes involved in heparan sulphate biosynthesis are associated with Plasmodium falciparum parasitaemia: a familial study in Burkina Faso. Malar J 2012, 11:108.

32. Coppi A, Tewari R, Bishop JR, Bennett BL, Lawrence R, Esko JD, Billker O, Sinnis P: Heparan sulfate proteoglycans provide a signal to Plasmodium sporozoites to stop migrating and productively invade host cells. Cell Host Microbe 2007, 2:316-327.

doi:10.1186/1475-2875-13-198

Cite this article as: Brisebarre et al:: A genome scan for Plasmodium falciparum malaria identifies quantitative trait loci on chromosomes 5q31, 6p21.3, 17p12, and 19p13. Malaria Journal 2014 13:198.

\section{Submit your next manuscript to BioMed Central and take full advantage of:}

- Convenient online submission

- Thorough peer review

- No space constraints or color figure charges

- Immediate publication on acceptance

- Inclusion in PubMed, CAS, Scopus and Google Scholar

- Research which is freely available for redistribution

Submit your manuscript at www.biomedcentral.com/submit
(O) Biomed Central 